\title{
Holes and Islands in Random Point Sets
}

\section{Conference Paper}

Author(s):

Balko, Martin; Scheucher, Manfred; Valtr, Pavel

Publication date:

2020

Permanent link:

https://doi.org/10.3929/ethz-b-000421477

Rights / license:

Creative Commons Attribution 3.0 Unported

Originally published in:

Leibniz International Proceedings in Informatics (LIPIcs) 164, https://doi.org/10.4230/LIPIcs.SoCG.2020.14 


\title{
Holes and Islands in Random Point Sets
}

\author{
Martin Balko \\ Department of Applied Mathematics, Faculty of Mathematics and Physics, \\ Charles University, Prague, Czech Republic \\ balko@kam.mff.cuni.cz
}

\section{Manfred Scheucher}

Institut für Mathematik, Technische Universität Berlin, Germany

scheucher@math.tu-berlin.de

\section{Pavel Valtr}

Department of Applied Mathematics, Faculty of Mathematics and Physics, Charles University, Prague, Czech Republic

Department of Computer Science, ETH Zürich, Switzerland

\begin{abstract}
For $d \in \mathbb{N}$, let $S$ be a finite set of points in $\mathbb{R}^{d}$ in general position. A set $H$ of $k$ points from $S$ is a $k$-hole in $S$ if all points from $H$ lie on the boundary of the convex hull $\operatorname{conv}(H)$ of $H$ and the interior of $\operatorname{conv}(H)$ does not contain any point from $S$. A set $I$ of $k$ points from $S$ is a $k$-island in $S$ if $\operatorname{conv}(I) \cap S=I$. Note that each $k$-hole in $S$ is a $k$-island in $S$.

For fixed positive integers $d, k$ and a convex body $K$ in $\mathbb{R}^{d}$ with $d$-dimensional Lebesgue measure 1 , let $S$ be a set of $n$ points chosen uniformly and independently at random from $K$. We show that the expected number of $k$-islands in $S$ is in $O\left(n^{d}\right)$. In the case $k=d+1$, we prove that the expected number of empty simplices (that is, $(d+1)$-holes) in $S$ is at most $2^{d-1} \cdot d ! \cdot\left(\begin{array}{l}n \\ d\end{array}\right)$. Our results improve and generalize previous bounds by Bárány and Füredi [4], Valtr [19], Fabila-Monroy and Huemer [8], and Fabila-Monroy, Huemer, and Mitsche [9].
\end{abstract}

2012 ACM Subject Classification Mathematics of computing $\rightarrow$ Combinatoric problems; Theory of computation $\rightarrow$ Computational geometry

Keywords and phrases stochastic geometry, random point set, Erdős-Szekeres type problem, $k$-hole, $k$-island, empty polytope, convex position, Horton set

Digital Object Identifier 10.4230/LIPIcs.SoCG.2020.14

Related Version A full version of this paper is available at https://arxiv.org/abs/2003.00909.

Funding Martin Balko: was supported by the grant no. 18-19158S of the Czech Science Foundation (GAČR), by the Center for Foundations of Modern Computer Science (Charles University project $\mathrm{UNCE} / \mathrm{SCI} / 004)$, and by the PRIMUS/17/SCI/3 project of Charles University. This article is part of a project that has received funding from the European Research Council (ERC) under the European Union's Horizon 2020 research and innovation programme (grant agreement No 810115). Manfred Scheucher: was supported by DFG Grant FE 340/12-1.

Pavel Valtr: was supported by the grant no. 18-19158S of the Czech Science Foundation (GAČR) and by the PRIMUS/17/SCI/3 project of Charles University.

\section{Introduction}

For $d \in \mathbb{N}$, let $S$ be a finite set of points in $\mathbb{R}^{d}$. The set $S$ is in general position if, for every $k=1, \ldots, d-1$, no $k+2$ points of $S$ lie in an affine $k$-dimensional subspace. A set $H$ of $k$ points from $S$ is a $k$-hole in $S$ if $H$ is in convex position and the interior of the convex hull $\operatorname{conv}(H)$ of $H$ does not contain any point from $S$; see Figure 1 for an illustration in the plane. We say that a subset of $S$ is a hole in $S$ if it is a $k$-hole in $S$ for some integer $k$.

(c) (i) (i) Martin Balko, Manfred Scheucher, and Pavel Valtr;

36th International Symposium on Computational Geometry (SoCG 2020).

Editors: Sergio Cabello and Danny Z. Chen; Article No. 14; pp. 14:1-14:16

Leibniz International Proceedings in Informatics

LI I ICS Schloss Dagstuhl - Leibniz-Zentrum für Informatik, Dagstuhl Publishing, Germany 
(a)

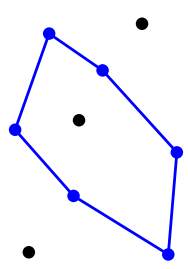

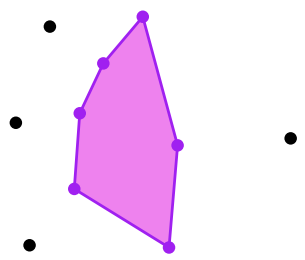

(b)

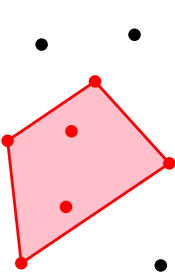

(c)

Figure 1 (a) A 6-tuple of points in convex position in a planar set $S$ of 10 points. (b) A 6-hole in $S$. (c) A 6 -island in $S$ whose points are not in convex position.

Let $h(k)$ be the smallest positive integer $N$ such that every set of $N$ points in general position in the plane contains a $k$-hole. In the 1970s, Erdős [6] asked whether the number $h(k)$ exists for every $k \in \mathbb{N}$. It was shown in the 1970 s and 1980 s that $h(4)=5, h(5)=10$ [11], and that $h(k)$ does not exist for every $k \geq 7$ [12]. That is, while every sufficiently large set contains a 4-hole and a 5-hole, Horton constructed arbitrarily large sets with no 7-holes. His construction was generalized to so-called Horton sets by Valtr [18]. The existence of 6-holes in every sufficiently large point set remained open until 2007, when Gerken [10] and Nicolas [15] independently showed that $h(6)$ exists; see also [20].

These problems were also considered in higher dimensions. For $d \geq 2$, let $h_{d}(k)$ be the smallest positive integer $N$ such that every set of $N$ points in general position in $\mathbb{R}^{d}$ contains a $k$-hole. In particular, $h_{2}(k)=h(k)$ for every $k$. Valtr [18] showed that $h_{d}(k)$ exists for $k \leq 2 d+1$ but it does not exist for $k>2^{d-1}(P(d-1)+1)$, where $P(d-1)$ denotes the product of the first $d-1$ prime numbers. The latter result was obtained by constructing multidimensional analogues of the Horton sets.

After the existence of $k$-holes was settled, counting the minimum number $H_{k}(n)$ of $k$-holes in any set of $n$ points in the plane in general position attracted a lot of attention. It is known, and not difficult to show, that $H_{3}(n)$ and $H_{4}(n)$ are in $\Omega\left(n^{2}\right)$. The currently best known lower bounds on $H_{3}(n)$ and $H_{4}(n)$ were proved in [1]. The best known upper bounds are due to Bárány and Valtr [5]. Altogether, these estimates are

$$
n^{2}+\Omega\left(n \log ^{2 / 3} n\right) \leq H_{3}(n) \leq 1.6196 n^{2}+o\left(n^{2}\right)
$$

and

$$
\frac{n^{2}}{2}+\Omega\left(n \log ^{3 / 4} n\right) \leq H_{4}(n) \leq 1.9397 n^{2}+o\left(n^{2}\right) .
$$

For $H_{5}(n)$ and $H_{6}(n)$, the best quadratic upper bounds can be found in [5]. The best lower bounds, however, are only $H_{5}(n) \geq \Omega\left(n \log ^{4 / 5} n\right)[1]$ and $H_{6}(n) \geq \Omega(n)$ [21]. For more details, we also refer to the second author's dissertation [17].

The quadratic upper bound on $H_{3}(n)$ can be also obtained using random point sets. For $d \in \mathbb{N}$, a convex body in $\mathbb{R}^{d}$ is a compact convex set in $\mathbb{R}^{d}$ with a nonempty interior. Let $k$ be a positive integer and let $K \subseteq \mathbb{R}^{d}$ be a convex body with $d$-dimensional Lebesgue measure $\lambda_{d}(K)=1$. We use $E H_{d, k}^{K}(n)$ to denote the expected number of $k$-holes in sets of $n$ points chosen independently and uniformly at random from $K$. The quadratic upper bound on $H_{3}(n)$ then also follows from the following bound of Bárány and Füredi [4] on the expected number of $(d+1)$-holes:

$$
E H_{d, d+1}^{K}(n) \leq(2 d)^{2 d^{2}} \cdot\left(\begin{array}{l}
n \\
d
\end{array}\right)
$$


for any $d$ and $K$. In the plane, Bárány and Füredi [4] proved $E H_{2,3}^{K}(n) \leq 2 n^{2}+O(n \log n)$ for every $K$. This bound was later slightly improved by Valtr [19], who showed $E H_{2,3}^{K}(n) \leq 4\left(\begin{array}{c}n \\ 2\end{array}\right)$ for any $K$. In the other direction, every set of $n$ points in $\mathbb{R}^{d}$ in general position contains at least $\left(\begin{array}{c}n-1 \\ d\end{array}\right)(d+1)$-holes $[4,13]$.

The expected number $E H_{2,4}^{K}(n)$ of 4-holes in random sets of $n$ points in the plane was considered by Fabila-Monroy, Huemer, and Mitsche [9], who showed

$$
E H_{2,4}^{K}(n) \leq 18 \pi D^{2} n^{2}+o\left(n^{2}\right)
$$

for any $K$, where $D=D(K)$ is the diameter of $K$. Since we have $D \geq 2 / \sqrt{\pi}$, by the Isodiametric inequality [7], the leading constant in (2) is at least 72 for any $K$.

In this paper, we study the number of $k$-holes in random point sets in $\mathbb{R}^{d}$. In particular, we obtain results that imply quadratic upper bounds on $H_{k}(n)$ for any fixed $k$ and that both strengthen and generalize the bounds by Bárány and Füredi [4], Valtr [19], and Fabila-Monroy, Huemer, and Mitsche [9].

\section{Our results}

Throughout the whole paper we only consider point sets in $\mathbb{R}^{d}$ that are finite and in general position.

\subsection{Islands and holes in random point sets}

First, we prove a result that gives the estimate $O\left(n^{d}\right)$ on the minimum number of $k$-holes in a set of $n$ points in $\mathbb{R}^{d}$ for any fixed $d$ and $k$. In fact, we prove the upper bound $O\left(n^{d}\right)$ even for so-called $k$-islands, which are also frequently studied in discrete geometry. A set $I$ of $k$ points from a point set $S \subseteq \mathbb{R}^{d}$ is a $k$-island in $S$ if $\operatorname{conv}(I) \cap S=I$; see part (c) of Figure 1 . Note that $k$-holes in $S$ are exactly those $k$-islands in $S$ that are in convex position. A subset of $S$ is an island in $S$ if it is a $k$-island in $S$ for some integer $k$.

- Theorem 1. Let $d \geq 2$ and $k \geq d+1$ be integers and let $K$ be a convex body in $\mathbb{R}^{d}$ with $\lambda_{d}(K)=1$. If $S$ is a set of $n \geq k$ points chosen uniformly and independently at random from $K$, then the expected number of $k$-islands in $S$ is at most

$$
2^{d-1} \cdot\left(2 d^{2 d-1}\left(\begin{array}{c}
k \\
\lfloor d / 2\rfloor
\end{array}\right)\right)^{k-d-1} \cdot(k-d) \cdot \frac{n(n-1) \cdots(n-k+2)}{(n-k+1)^{k-d-1}},
$$

which is in $O\left(n^{d}\right)$ for any fixed $d$ and $k$.

The bound in Theorem 1 is tight up to a constant multiplicative factor that depends on $d$ and $k$, as, for any fixed $k \geq d$, every set $S$ of $n$ points in $\mathbb{R}^{d}$ in general position contains at least $\Omega\left(n^{d}\right) k$-islands. To see this, observe that any $d$-tuple $T$ of points from $S$ determines a $k$-island with $k-d$ closest points to the hyperplane spanned by $T$ (ties can be broken by, for example, taking points with lexicographically smallest coordinates), as $S$ is in general position and thus $T$ is a $d$-hole in $S$. Any such $k$-tuple of points from $S$ contains $\left(\begin{array}{l}k \\ d\end{array}\right) d$-tuples of points from $S$ and thus we have at least $\left(\begin{array}{l}n \\ d\end{array}\right) /\left(\begin{array}{l}k \\ d\end{array}\right) \in \Omega\left(n^{d}\right) k$-islands in $S$.

Thus, by Theorem 1 , random point sets in $\mathbb{R}^{d}$ asymptotically achieve the minimum number of $k$-islands. This is in contrast with the fact that, unlike Horton sets, they contain arbitrarily large holes. Quite recently, Balogh, González-Aguilar, and Salazar [3] showed that the expected number of vertices of the largest hole in a set of $n$ random points chosen independently and uniformly over a convex body in the plane is in $\Theta(\log n /(\log \log n))$.

For $k$-holes, we modify the proof of Theorem 1 to obtain a slightly better estimate. 
- Theorem 2. Let $d \geq 2$ and $k \geq d+1$ be integers and let $K$ be a convex body in $\mathbb{R}^{d}$ with $\lambda_{d}(K)=1$. If $S$ is a set of $n \geq k$ points chosen uniformly and independently at random from $K$, then the expected number $E H_{d, k}^{K}(n)$ of $k$-holes in $S$ is in $O\left(n^{d}\right)$ for any fixed $d$ and $k$. More precisely,

$$
E H_{d, k}^{K}(n) \leq 2^{d-1} \cdot\left(2 d^{2 d-1}\left(\begin{array}{c}
k \\
\lfloor d / 2\rfloor
\end{array}\right)\right)^{k-d-1} \cdot \frac{n(n-1) \cdots(n-k+2)}{(k-d-1) ! \cdot(n-k+1)^{k-d-1}} .
$$

For $d=2$ and $k=4$, Theorem 2 implies $E H_{2,4}^{K}(n) \leq 128 \cdot n^{2}+o\left(n^{2}\right)$ for any $K$, which is a worse estimate than (2) if the diameter of $K$ is at most $8 /(3 \sqrt{\pi}) \simeq 1.5$. However, the proof of Theorem 2 can be modified to give $E H_{2,4}^{K}(n) \leq 12 n^{2}+o\left(n^{2}\right)$ for any $K$, which is always better than (2); see the final remarks in Section 3. We believe that the leading constant in $E H_{2,4}^{K}(n)$ can be estimated even more precisely and we hope to discuss this direction in future work.

In the case $k=d+1$, the bound in Theorem 2 simplifies to the following estimate on the expected number of $(d+1)$-holes (also called empty simplices) in random sets of $n$ points in $\mathbb{R}^{d}$.

- Corollary 3. Let $d \geq 2$ be an integer and let $K$ be a convex body in $\mathbb{R}^{d}$ with $\lambda_{d}(K)=1$. If $S$ is a set of $n$ points chosen uniformly and independently at random from $K$, then the expected number of $(d+1)$-holes in $S$ satisfies

$$
E H_{d, d+1}^{K}(n) \leq 2^{d-1} \cdot d ! \cdot\left(\begin{array}{l}
n \\
d
\end{array}\right) .
$$

Corollary 3 is stronger than the bound (1) by Bárány and Füredi [4] and, in the planar case, coincides with the bound $E H_{2,3}^{K}(n) \leq 4\left(\begin{array}{c}n \\ 2\end{array}\right)$ by Valtr [19]. Very recently, Reitzner and Temesvari [16] proved an upper bound on $E H_{d, d+1}^{K}(n)$ that is asymptotically tight if $d=2$ or if $d \geq 3$ and $K$ is an ellipsoid. In the planar case, their result shows that the bound $4\left(\begin{array}{c}n \\ 2\end{array}\right)$ on $E H_{2,3}^{K}(n)$ is best possible, up to a smaller order error term. No tight bounds on $E H_{d, d+1}^{K}(n)$ are known if $d \geq 3$ and $K$ is not an ellipsoid.

We also consider islands of all possible sizes and show that their expected number is in $2^{\Theta\left(n^{(d-1) /(d+1)}\right)}$.

- Theorem 4. Let $d \geq 2$ be an integer and let $K$ be a convex body in $\mathbb{R}^{d}$ with $\lambda_{d}(K)=1$. Then there are constants $C_{1}=C_{1}(d), C_{2}=C_{2}(d)$, and $n_{0}=n_{0}(d)$ such that for every set $S$ of $n \geq n_{0}$ points chosen uniformly and independently at random from $K$ the expected number $E_{d}^{K}$ of islands in $S$ satisfies

$$
2^{C_{1} \cdot n^{(d-1) /(d+1)}} \leq E_{d}^{K} \leq 2^{C_{2} \cdot n^{(d-1) /(d+1)}} .
$$

Since each island in $S$ has at most $n$ points, there is a $k \in\{1, \ldots, n\}$ such that the expected number of $k$-islands in $S$ is at least $(1 / n)$-fraction of the expected number of all islands, which is still in $2^{\Omega\left(n^{(d-1) /(d+1)}\right)}$. This shows that the expected number of $k$-islands can become asymptotically much larger than $O\left(n^{d}\right)$ if $k$ is not fixed. Due to space limitations, the proof of Theorem 4 is omitted.

\subsection{Islands and holes in $d$-Horton sets}

To our knowledge, Theorem 1 is the first nontrivial upper bound on the minimum number of $k$-islands a point set in $\mathbb{R}^{d}$ with $d>2$ can have. For $d=2$, Fabila-Monroy and Huemer [8] showed that, for every fixed $k \in \mathbb{N}$, the Horton sets with $n$ points contain only $O\left(n^{2}\right)$ 
$k$-islands. For $d>2$, Valtr [18] introduced a $d$-dimensional analogue of Horton sets. Perhaps surprisingly, these sets contain asymptotically more than $O\left(n^{d}\right) k$-islands for $k \geq d+1$. For each $k$ with $d+1 \leq k \leq 3 \cdot 2^{d-1}$, they even contain asymptotically more than $O\left(n^{d}\right) k$-holes.

Theorem 5. Let $d \geq 2$ and $k$ be fixed positive integers. Then every d-dimensional Horton set $H$ with $n$ points contains at least $\Omega\left(n^{\min \left\{2^{d-1}, k\right\}}\right) k$-islands in $H$. If $k \leq 3 \cdot 2^{d-1}$, then $H$ even contains at least $\Omega\left(n^{\min \left\{2^{d-1}, k\right\}}\right) k$-holes in $H$.

\section{Proofs of Theorem 1 and Theorem 2}

Let $d$ and $k$ be positive integers and let $K$ be a convex body in $\mathbb{R}^{d}$ with $\lambda_{d}(K)=1$. Let $S$ be a set of $n$ points chosen uniformly and independently at random from $K$. Note that $S$ is in general position with probability 1 . We assume $k \geq d+1$, as otherwise the number of $k$-islands in $S$ is trivially $\left(\begin{array}{l}n \\ k\end{array}\right)$ in every set of $n$ points in $\mathbb{R}^{d}$ in general position. We also assume $d \geq 2$ and $n \geq k$, as otherwise the number of $k$-islands is trivially $n-k+1$ and 0 , respectively, in every set of $n$ points in $\mathbb{R}^{d}$.

First, we prove Theorem 1 by showing that the expected number of $k$-islands in $S$ is at most

$$
2^{d-1} \cdot\left(2 d^{2 d-1}\left(\begin{array}{c}
k \\
\lfloor d / 2\rfloor
\end{array}\right)\right)^{k-d-1} \cdot(k-d) \cdot \frac{n(n-1) \cdots(n-k+2)}{(n-k+1)^{k-d-1}}
$$

which is in $O\left(n^{d}\right)$ for any fixed $d$ and $k$. At the end of this section, we improve the bound for $k$-holes, which will prove Theorem 2 .

Let $Q$ be a set of $k$ points from $S$. We first introduce a suitable unique ordering $q_{1}, \ldots, q_{k}$ of points from $Q$. First, we take a set $D$ of $d+1$ points from $Q$ that determine a simplex $\Delta$ with largest volume among all $(d+1)$-tuples of points from $Q$. Let $q_{1} q_{2}$ be the longest edge of $\Delta$ with $q_{1}$ lexicographically smaller than $q_{2}$ and let $a$ be the number of points from $Q$ inside $\Delta$. For every $i=2, \ldots, d$, let $q_{i+1}$ be the furthest point from $D \backslash\left\{q_{1}, \ldots, q_{i}\right\}$ to aff $\left(q_{1}, \ldots, q_{i}\right)$. Next, we let $q_{d+2}, \ldots, q_{d+a+1}$ be the $a$ points of $Q$ inside $\Delta$ ordered lexicographically. The remaining $k-d-a-1$ points $q_{d+a+2}, \ldots, q_{k}$ from $Q$ lie outside of $\Delta$ and we order them so that, for every $i=1, \ldots, k-a-d-1$, the point $q_{d+a+i+1}$ is closest to $\operatorname{conv}\left(\left\{q_{1}, \ldots\right.\right.$, $\left.\left.q_{d+a+i}\right\}\right)$ among the points $q_{d+a+i+1}, \ldots, q_{k}$. In case of a tie in any of the conditions, we choose the point with lexicographically smallest coordinates. Note, however, that a tie occurs with probability 0 .

Clearly, there is a unique such ordering $q_{1}, \ldots, q_{k}$ of $Q$. We call this ordering the canonical $(k, a)$-ordering of $Q$. To reformulate, an ordering $q_{1}, \ldots, q_{k}$ of $Q$ is the canonical $(k, a)$-ordering of $Q$ if and only if the following five conditions are satisfied:

(L1) The $d$-dimensional simplex $\Delta$, with vertices $q_{1}, \ldots, q_{d+1}$ has the largest $d$-dimensional Lebesgue measure among all $d$-dimensional simplices spanned by points from $Q$.

(L2) For every $i=1, \ldots, d-1$, the point $q_{i+1}$ has the largest distance among all points from $\left\{q_{i+1}, \ldots, q_{d}\right\}$ to the $(i-1)$-dimensional affine subspace aff $\left(q_{1}, \ldots, q_{i}\right)$ spanned by $q_{1}, \ldots, q_{i}$. Moreover, $q_{1}$ is lexicographically smaller than $q_{2}$.

(L3) For every $i=1, \ldots, d-1$, the distance between $q_{i+1}$ and aff $\left(q_{1}, \ldots, q_{i}\right)$ is at least as large as the distance between $q_{d+1}$ and aff $\left(q_{1}, \ldots, q_{i}\right)$. Also, the distance between $q_{1}$ and $q_{2}$ is at least as large as the distance between $q_{d+1}$ and any $q_{i}$ with $i \in\{1, \ldots, d\}$.

(L4) The points $q_{d+2}, \ldots, q_{d+a+1}$ lie inside $\Delta$ and are ordered lexicographically.

(L5) The points $q_{d+a+2}, \ldots, q_{k}$ lie outside of $\Delta$. For every $i=1, \ldots, k-a-d-1$, the point $q_{d+a+i+1}$ is closest to $\operatorname{conv}\left(\left\{q_{1}, \ldots, q_{d+a+i}\right\}\right)$ among the points $q_{d+a+i+1}, \ldots, q_{k}$. 
Figure 2 gives an illustration in $\mathbb{R}^{2}$. We note that the conditions (L2) and (L3) can be merged together. However, later in the proof, we use the fact that the probability that the points from $Q$ satisfy the condition (L2) equals $1 / d$ !, so we stated the two conditions separately.

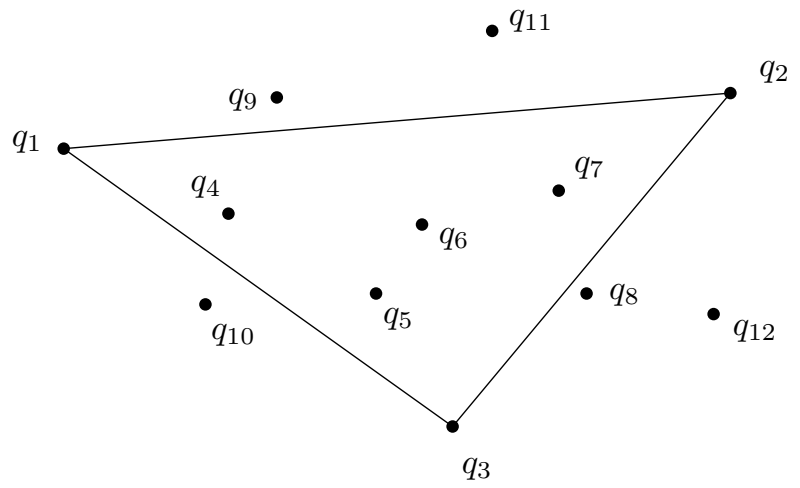

Figure 2 An illustration of the canonical $(k, a)$-ordering of a planar point set $Q$. Here we have $k=12$ points and $a=4$ of the points lie inside the largest area triangle $\triangle$ with vertices $q_{1}, q_{2}, q_{3}$.

Before going into details, we first give a high-level overview of the proof of Theorem 1. First, we prove an $O\left(1 / n^{a+1}\right)$ bound on the probability that $\triangle$ contains precisely the points $p_{d+2}, \ldots, p_{d+1+a}$ from $S$ (Lemma 9 ), which means that the points $p_{1}, \ldots, p_{d+1+a}$ determine an island in $S$. Next, for $i=d+2+a, \ldots, k$, we show that, conditioned on the fact that the $(i-1)$-tuple $\left(p_{1}, \ldots, p_{i-1}\right)$ determines an island in $S$ in the canonical $(k, a)$-ordering, the $i$-tuple $\left(p_{1}, \ldots, p_{i}\right)$ determines an island in $S$ in the canonical $(k, a)$-ordering with probability $O(1 / n)$ (Lemma 10). Then it immediately follows that the probability that $I$ determines a $k$-island in $S$ with the desired properties is at most $O\left(1 / n^{a+1} \cdot(1 / n)^{k-(d+1+a)}\right)=O\left(n^{d-k}\right)$. Since there are $n \cdot(n-1) \cdots(n-k+1)=O\left(n^{k}\right)$ possibilities to select such an ordered subset $I$ and each $k$-island in $S$ is counted at most $k$ ! times, we obtain the desired bound $O\left(n^{k} \cdot n^{d-k} \cdot k !\right)=O\left(n^{d}\right)$ on the expected number of $k$-islands in $S$.

We now proceed with the proof. Let $p_{1}, \ldots, p_{k}$ be points from $S$ in the order in which they are drawn from $K$. We use $\Delta$ to denote the $d$-dimensional simplex with vertices $p_{1}, \ldots, p_{d+1}$. We eventually show that the probability that $p_{1}, \ldots, p_{k}$ is the canonical $(k, a)$-ordering of a $k$-island in $S$ for some $a$ is at most $O\left(1 / n^{k-d}\right)$. First, however, we need to state some notation and prove some auxiliary results.

Consider the points $p_{1}, \ldots, p_{d}$. Without loss of generality, we can assume that, for each $i=1, \ldots, d$, the point $p_{i}$ has the last $d-i+1$ coordinates equal to zero. Otherwise we apply a suitable isometry to $S$. Then, for every $i=1, \ldots, d$, the distance between $p_{i+1}$ and the $(i-1)$-dimensional affine subspace spanned by $p_{1}, \ldots, p_{i}$ is equal to the absolute value of the $i$ th coordinate of $p_{i+1}$. Moreover, after applying a suitable rotation, we can also assume that the first coordinate of each of the points $p_{1}, \ldots, p_{d}$ is nonnegative.

Let $\Delta_{0}$ be the $(d-1)$-dimensional simplex with vertices $p_{1}, \ldots, p_{d}$ and let $H$ be the hyperplane containing $\Delta_{0}$. Note that, according to our assumptions about $p_{1}, \ldots, p_{d}$, we have $H=\left\{\left(x_{1}, \ldots, x_{d}\right) \in \mathbb{R}^{d}: x_{d}=0\right\}$. Let $B$ be the set of points $\left(x_{1}, \ldots, x_{d}\right) \in \mathbb{R}^{d}$ that satisfy the following three conditions:

(i) $x_{1} \geq 0$,

(ii) $\left|x_{i}\right|$ is at most as large as the absolute value of the $i$ th coordinate of $p_{i+1}$ for every $i \in\{1, \ldots, d-1\}$, and

(iii) $\left|x_{d}\right| \leq d / \lambda_{d-1}\left(\Delta_{0}\right)$. 
See Figures $3 \mathrm{a}$ and $3 \mathrm{~b}$ for illustrations in $\mathbb{R}^{2}$ and $\mathbb{R}^{3}$, respectively. Observe that $B$ is a $d$-dimensional axis-parallel box. For $h \in \mathbb{R}$, we use $I_{h}$ to denote the intersection of $B$ with the hyperplane $x_{d}=h$.

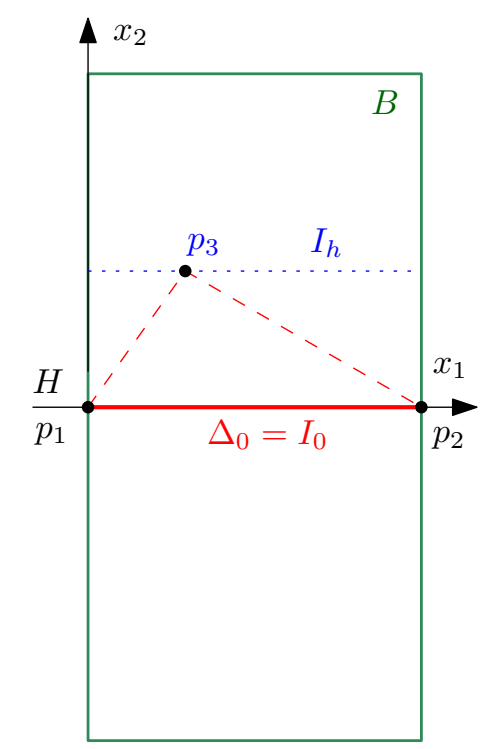

(a)

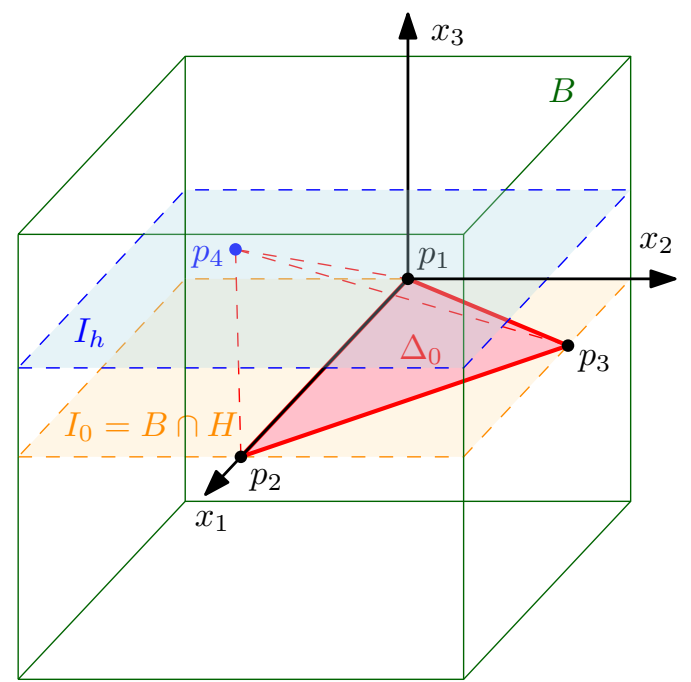

(b)

Figure 3 An illustration of the proof of Theorem 1 in (a) $\mathbb{R}^{2}$ and (b) $\mathbb{R}^{3}$.

Having fixed $p_{1}, \ldots, p_{d}$, we now try to restrict possible locations of the points $p_{d+1}, \ldots, p_{k}$, one by one, so that $p_{1}, \ldots, p_{k}$ is the canonical $(k, a)$-ordering of a $k$-island in $S$ for some $a$. First, we observe that the position of the point $p_{d+1}$ is restricted to $B$.

- Lemma 6. If $p_{1}, \ldots, p_{d+1}$ satisfy condition (L3), then $p_{d+1}$ lies in the box $B$.

Proof. Let $p_{d+1}=\left(x_{1}, \ldots, x_{d}\right)$. According to our choice of points $p_{1}, \ldots, p_{d}$ and from the assumption that $p_{1}, \ldots, p_{d}$ satisfy (L3), we get $x_{1} \geq 0$ and also that $\left|x_{i}\right|$ is at most as large as the absolute value of the $i$ th coordinate of $p_{i+1}$ for every $i \in\{1, \ldots, d-1\}$.

It remains to show that $\left|x_{d}\right| \leq d / \lambda_{d-1}\left(\Delta_{0}\right)$. The simplex $\Delta$ spanned by $p_{1}, \ldots, p_{d+1}$ is contained in the convex body $K$, as $p_{1}, \ldots, p_{d+1} \in K$ and $K$ is convex. Thus $\lambda_{d}(\Delta) \leq$ $\lambda_{d}(K)=1$. On the other hand, the volume $\lambda_{d}(\Delta)$ equals $\lambda_{d-1}\left(\Delta_{0}\right) \cdot h / d$, where $h$ is the distance between $p_{d+1}$ and the hyperplane $H$ containing $\Delta_{0}$. According to our assumptions about $p_{1}, \ldots, p_{d}$, the distance $h$ equals $\left|x_{d}\right|$. Since $\lambda_{d}(\Delta) \leq 1$, it follows that $\left|x_{d}\right|=h \leq$ $d / \lambda_{d-1}\left(\Delta_{0}\right)$ and thus $p_{d+1} \in B$.

The following auxiliary lemma gives an identity that is needed later. We omit the proof, which can be found, for example, in [2, Section 1].

- Lemma 7 ([2]). For all nonnegative integers a and b, we have

$$
\int_{0}^{1} x^{a}(1-x)^{b} \mathrm{~d} x=\frac{a ! b !}{(a+b+1) !} .
$$

We will also use the following result, called the Asymptotic Upper Bound Theorem [14], that estimates the maximum number of facets in a polytope. 
- Theorem 8 (Asymptotic Upper Bound Theorem [14]). For every integer $d \geq 2$, a $d$ dimensional convex polytope with $N$ vertices has at most $2\left(\begin{array}{c}N \\ \lfloor d / 2\rfloor\end{array}\right)$ facets.

Let $a$ be an integer satisfying $0 \leq a \leq k-d-1$ and let $E_{a}$ be the event that $p_{1}, \ldots, p_{k}$ is the canonical $(k, a)$-ordering such that $\left\{p_{1}, \ldots, p_{d+a+1}\right\}$ is an island in $S$. To estimate the probability that $p_{1}, \ldots, p_{k}$ is the canonical $(k, a)$-ordering of a $k$-island in $S$, we first find an upper bound on the conditional probability of $E_{a}$, conditioned on the event $L_{2}$ that $p_{1}, \ldots, p_{d}$ satisfy (L2).

- Lemma 9. For every $a \in\{0, \ldots, k-d-1\}$, the probability $\operatorname{Pr}\left[E_{a} \mid L_{2}\right]$ is at most

$$
\frac{2^{d-1} \cdot d !}{(k-a-d-1) ! \cdot(n-k+1)^{a+1}} .
$$

Proof. It follows from Lemma 6 that, in order to satisfy (L3), the point $p_{d+1}$ must lie in the box $B$. In particular, $p_{d+1}$ is contained in $I_{h} \cap K$ for some real number $h \in$ $\left[-d / \lambda_{d-1}\left(\Delta_{0}\right), d / \lambda_{d-1}\left(\Delta_{0}\right)\right]$. If $p_{d+1} \in I_{h}$, then the simplex $\Delta=\operatorname{conv}\left(\left\{p_{1}, \ldots, p_{d+1}\right\}\right)$ has volume $\lambda_{d}(\Delta)=\lambda_{d-1}\left(\Delta_{0}\right) \cdot|h| / d$ and the $a$ points $p_{d+2}, \ldots, p_{d+a+1}$ satisfy (L4) with probability

$$
\frac{1}{a !} \cdot\left(\lambda_{d}(\Delta)\right)^{a}=\frac{1}{a !} \cdot\left(\frac{\lambda_{d-1}\left(\Delta_{0}\right) \cdot|h|}{d}\right)^{a},
$$

as they all lie in $\Delta \subseteq K$ in the unique order.

In order to satisfy the condition (L5), the $k-a-d-1$ points $p_{d+a+i+1}$, for $i \in$ $\{1, \ldots, k-a-d-1\}$, must have increasing distance to $\operatorname{conv}\left(\left\{p_{1}, \ldots, p_{d+a+i}\right\}\right)$ as the index $i$ increases, which happens with probability at most $\frac{1}{(k-a-d-1) !}$. Since $\left\{p_{1}, \ldots, p_{d+a+1}\right\}$ must be an island in $S$, the $n-d-a-1$ points from $S \backslash\left\{p_{1}, \ldots, p_{d+a+1}\right\}$ must lie outside $\Delta$. If $p_{d+1} \in I_{h}$, then this happens with probability

$$
\left(\lambda_{d}(K \backslash \Delta)\right)^{n-d-a-1}=\left(\lambda_{d}(K)-\lambda_{d}(\Delta)\right)^{n-d-a-1}=\left(1-\frac{\lambda_{d-1}\left(\Delta_{0}\right) \cdot|h|}{d}\right)^{n-d-a-1},
$$

as they all lie in $K \backslash \Delta$ and we have $\Delta \subseteq K$ and $\lambda_{d}(K)=1$.

Altogether, we get that $\operatorname{Pr}\left[E_{a} \mid L_{2}\right]$ is at most

$$
\int_{-d / \lambda_{d-1}\left(\Delta_{0}\right)}^{d / \lambda_{d-1}\left(\Delta_{0}\right)} \frac{\lambda_{d-1}\left(I_{h} \cap K\right)}{a ! \cdot(k-a-d-1) !} \cdot\left(\frac{\lambda_{d-1}\left(\Delta_{0}\right) \cdot|h|}{d}\right)^{a} \cdot\left(1-\frac{\lambda_{d-1}\left(\Delta_{0}\right) \cdot|h|}{d}\right)^{n-d-a-1} \mathrm{~d} h .
$$

Since we have $\lambda_{d-1}\left(I_{0}\right)=\lambda_{d-1}\left(I_{h}\right)$ for every $h \in\left[-d / \lambda_{d-1}\left(\Delta_{0}\right), d / \lambda_{d-1}\left(\Delta_{0}\right)\right]$, we obtain $\lambda_{d-1}\left(I_{h} \cap K\right) \leq \lambda_{d-1}\left(I_{0}\right)$ and thus $\operatorname{Pr}\left[E_{a} \mid L_{2}\right]$ is at most

$$
\frac{2 \cdot \lambda_{d-1}\left(I_{0}\right)}{a ! \cdot(k-a-d-1) !} \cdot \int_{0}^{d / \lambda_{d-1}\left(\Delta_{0}\right)}\left(\frac{\lambda_{d-1}\left(\Delta_{0}\right) \cdot h}{d}\right)^{a} \cdot\left(1-\frac{\lambda_{d-1}\left(\Delta_{0}\right) \cdot h}{d}\right)^{n-d-a-1} \mathrm{~d} h .
$$

By substituting $t=\frac{\lambda_{d-1}\left(\Delta_{0}\right) \cdot h}{d}$, we obtain

$$
\operatorname{Pr}\left[E_{a} \mid L_{2}\right] \leq \frac{2 d \cdot \lambda_{d-1}\left(I_{0}\right)}{a ! \cdot(k-a-d-1) ! \cdot \lambda_{d-1}\left(\Delta_{0}\right)} \cdot \int_{0}^{1} t^{a}(1-t)^{n-d-a-1} \mathrm{~d} t .
$$


By Lemma 7, the right side in the above inequality equals

$$
\begin{aligned}
\frac{2 d \cdot \lambda_{d-1}\left(I_{0}\right)}{a ! \cdot(k-a-d-1) ! \cdot \lambda_{d-1}\left(\Delta_{0}\right)} \cdot & \frac{a ! \cdot(n-d-a-1) !}{(n-d) !} \\
& =\frac{2 d \cdot \lambda_{d-1}\left(I_{0}\right)}{(k-a-d-1) ! \cdot \lambda_{d-1}\left(\Delta_{0}\right)} \cdot \frac{(n-d-a-1) !}{(n-d) !} .
\end{aligned}
$$

For every $i=1, \ldots, d-1$, let $h_{i}$ be the distance between the point $p_{i+1}$ and the $(i-1)$ dimensional affine subspace spanned by $p_{1}, \ldots, p_{i}$. Since the volume of the box $I_{0}$ satisfies

$$
\lambda_{d-1}\left(I_{0}\right)=h_{1}\left(2 h_{2}\right) \cdots\left(2 h_{d-1}\right)=2^{d-2} \cdot h_{1} \cdots h_{d-1}
$$

and the volume of the $(d-1)$-dimensional simplex $\Delta_{0}$ is

$$
\lambda_{d-1}\left(\Delta_{0}\right)=\frac{h_{1}}{1} \cdot \frac{h_{2}}{2} \cdots \cdot \frac{h_{d-1}}{d-1}=\frac{h_{1} \cdots h_{d-1}}{(d-1) !}
$$

we obtain $\lambda_{d-1}\left(I_{0}\right) / \lambda_{d-1}\left(\Delta_{0}\right)=2^{d-2} \cdot(d-1)$ !. Thus

$$
\begin{aligned}
\operatorname{Pr}\left[E_{a} \mid L_{2}\right] & \leq \frac{2^{d-1} \cdot d !}{(k-a-d-1) !} \cdot \frac{(n-d-a-1) !}{(n-d) !} \\
& =\frac{2^{d-1} \cdot d !}{(k-a-d-1) ! \cdot(n-d) \cdots(n-d-a)} \\
& \leq \frac{2^{d-1} \cdot d !}{(k-a-d-1) ! \cdot(n-k+1)^{a+1}},
\end{aligned}
$$

where the last inequality follows from $a \leq k-d-1$.

For every $i \in\{d+a+1, \ldots, k\}$, let $E_{a, i}$ be the event that $p_{1}, \ldots, p_{k}$ is the canonical $(k, a)$-ordering such that $\left\{p_{1}, \ldots, p_{i}\right\}$ is an island in $S$. Note that in the event $E_{a, i}$ the condition (L5) implies that $\left\{p_{1}, \ldots, p_{j}\right\}$ is an island in $S$ for every $j \in\{d+a+1, \ldots, i\}$. Thus we have

$$
L_{2} \supseteq E_{a}=E_{a, d+a+1} \supseteq E_{a, d+a+2} \supseteq \cdots \supseteq E_{a, k} .
$$

Moreover, the event $E_{a, k}$ says that $p_{1}, \ldots, p_{k}$ is the canonical $(k, a)$-ordering of a $k$-island in $S$. For $i \in\{d+a+2, \ldots, k\}$, we now estimate the conditional probability of $E_{a, i}$, conditioned on $E_{a, i-1}$.

- Lemma 10. For every $i \in\{d+a+2, \ldots, k\}$, we have

$$
\operatorname{Pr}\left[E_{a, i} \mid E_{a, i-1}\right] \leq \frac{2 d^{2 d-1} \cdot\left(\begin{array}{c}
k \\
\lfloor d / 2\rfloor
\end{array}\right)}{n-i+1} .
$$

Proof. Let $i \in\{d+a+2, \ldots, k\}$ and assume that the event $E_{a, i-1}$ holds. That is, $p_{1}, \ldots, p_{k}$ is the canonical $(k, a)$-ordering such that $\left\{p_{1}, \ldots, p_{i-1}\right\}$ is an $(i-1)$-island in $S$.

First, we assume that $\Delta$ is a regular simplex with height $\eta>0$. At the end of the proof we show that the case when $\Delta$ is an arbitrary simplex follows by applying a suitable affine transformation.

For every $j \in\{1, \ldots, d+1\}$, let $F_{j}$ be the facet $\operatorname{conv}\left(\left\{p_{1}, \ldots, p_{d+1}\right\} \backslash\left\{p_{j}\right\}\right)$ of $\Delta$ and let $H_{j}$ be the hyperplane parallel to $F_{j}$ that contains $p_{j}$. We use $H_{j}^{+}$to denote the halfspace determined by $H_{j}$ such that $\Delta \subseteq H_{j}^{+}$. We set $\Delta^{*}=\cap_{j=1}^{d+1} H_{j}^{+}$; see Figures $4 \mathrm{a}$ and $4 \mathrm{~b}$ for illustrations in $\mathbb{R}^{2}$ and $\mathbb{R}^{3}$, respectively. Note that $\Delta^{*}$ is a $d$-dimensional simplex containing $\Delta$. Also, notice that if $x \notin \Delta^{*}$, then $x \notin H_{j}^{+}$for some $j$ and the distance between $x$ and the hyperplane containing $F_{j}$ is larger than $\eta$. 


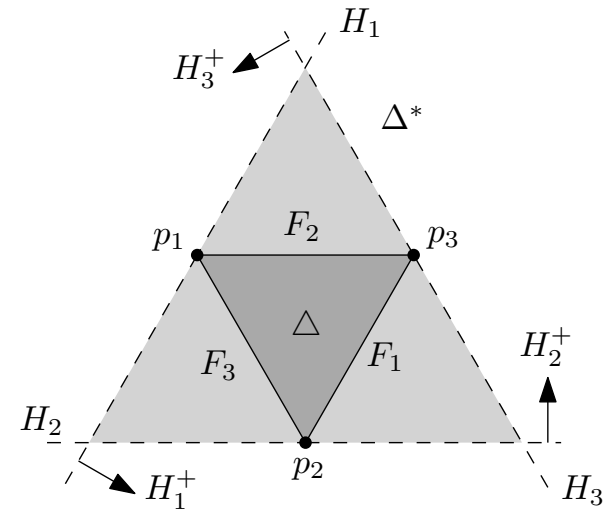

(a)

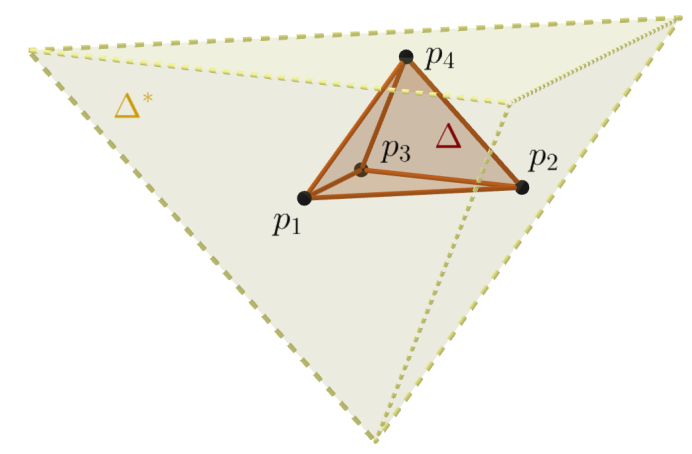

(b)

Figure 4 An illustration of (a) the simplex $\Delta^{*}$ in $\mathbb{R}^{2}$ and (b) in $\mathbb{R}^{3}$.

We show that the fact that $p_{1}, \ldots, p_{k}$ is the canonical $(k, a)$-ordering implies that every point from $\left\{p_{1}, \ldots, p_{k}\right\}$ is contained in $\Delta^{*}$. Suppose for contradiction that some point $p \in\left\{p_{1}, \ldots, p_{k}\right\}$ does not lie inside $\Delta^{*}$. Then there is a facet $F_{j}$ of $\Delta$ such that the distance $\eta^{\prime}$ between $p$ and the hyperplane containing $F_{j}$ is larger than $\eta$. Then, however, the simplex $\Delta^{\prime}$ spanned by vertices of $F_{j}$ and by $p$ has volume larger than $\Delta$, because

$$
\lambda_{d}\left(\Delta^{\prime}\right)=\frac{1}{d} \cdot \lambda_{d-1}\left(F_{j}\right) \cdot \eta^{\prime}>\frac{1}{d} \cdot \lambda_{d-1}\left(F_{j}\right) \cdot \eta=\lambda_{d}(\Delta) .
$$

This contradicts the fact that $p_{1}, \ldots, p_{k}$ is the canonical $(k, a)$-ordering, as, according to (L1), $\Delta$ has the largest $d$-dimensional Lebesgue measure among all $d$-dimensional simplices spanned by points from $\left\{p_{1}, \ldots, p_{k}\right\}$.

Let $\sigma$ be the barycenter of $\Delta$. For every point $p \in \Delta^{*} \backslash \Delta$, the line segment $\sigma p$ intersects at least one facet of $\Delta$. For every $j \in\{1, \ldots, d+1\}$, we use $R_{j}$ to denote the set of points $p \in \Delta^{*} \backslash \Delta$ for which the line segment $\sigma p$ intersects the facet $F_{j}$ of $\Delta$. Observe that each set $R_{j}$ is convex and the sets $R_{1}, \ldots, R_{d+1}$ partition $\Delta^{*} \backslash \Delta$ (up to their intersection of $d$-dimensional Lebesgue measure 0 ); see Figure 5 for an illustration in the plane.

Consider the point $p_{i}$. Since $p_{1}, \ldots, p_{k}$ is the canonical $(k, a)$-ordering, the condition (L5) implies that $p_{i}$ lies outside of the polytope $\operatorname{conv}\left(\left\{p_{1}, \ldots, p_{i-1}\right\}\right)$. To bound the probability $\operatorname{Pr}\left[E_{a, i} \mid E_{a, i-1}\right]$, we need to estimate the probability that $\operatorname{conv}\left(\left\{p_{1}, \ldots, p_{i}\right\}\right) \backslash$ $\operatorname{conv}\left(\left\{p_{1}, \ldots, p_{i-1}\right\}\right)$ does not contain any point from $S \backslash\left\{p_{1}, \ldots, p_{i}\right\}$, conditioned on $E_{a, i-1}$. We know that $p_{i}$ lies in $\Delta^{*} \backslash \Delta$ and that $p_{i} \in R_{j}$ for some $j \in\{1, \ldots, d+1\}$.

Since $p_{i} \notin \operatorname{conv}\left(\left\{p_{1}, \ldots, p_{i-1}\right\}\right)$, there is a facet $\varphi$ of the polytope $\operatorname{conv}\left(\left\{p_{1}, \ldots, p_{i-1}\right\}\right)$ contained in the closure of $R_{j}$ such that $\sigma p_{i}$ intersects $\varphi$. Since $S$ is in general position with probability 1 , we can assume that $\varphi$ is a $(d-1)$-dimensional simplex. The point $p_{i}$ is contained in the convex set $C_{\varphi}$ that contains all points $c \in \mathbb{R}^{d}$ such that the line segment $\sigma c$ intersects $\varphi$. We use $H(0)$ to denote the hyperplane containing $\varphi$. For a positive $r \in \mathbb{R}$, let $H(r)$ be the hyperplane parallel to $H(0)$ at distance $r$ from $H(0)$ such that $H(r)$ is contained in the halfspace determined by $H(0)$ that does not contain $\operatorname{conv}\left(\left\{p_{1}, \ldots, p_{i-1}\right\}\right)$. Then we have $p_{i} \in H(h)$ for some positive $h \in \mathbb{R}$.

Since $p_{i} \in K$ and $\varphi \subseteq K$, the convexity of $K$ implies that the $\operatorname{simplex} \operatorname{conv}\left(\varphi \cup\left\{p_{i}\right\}\right)$ has volume $\lambda_{d}\left(\operatorname{conv}\left(\varphi \cup\left\{p_{i}\right\}\right)\right) \leq \lambda_{d}(K)=1$. Since $\lambda_{d}\left(\operatorname{conv}\left(\varphi \cup\left\{p_{i}\right\}\right)\right)=\lambda_{d-1}(\varphi) \cdot h / d$, we obtain $h \leq d / \lambda_{d-1}(\varphi)$. 


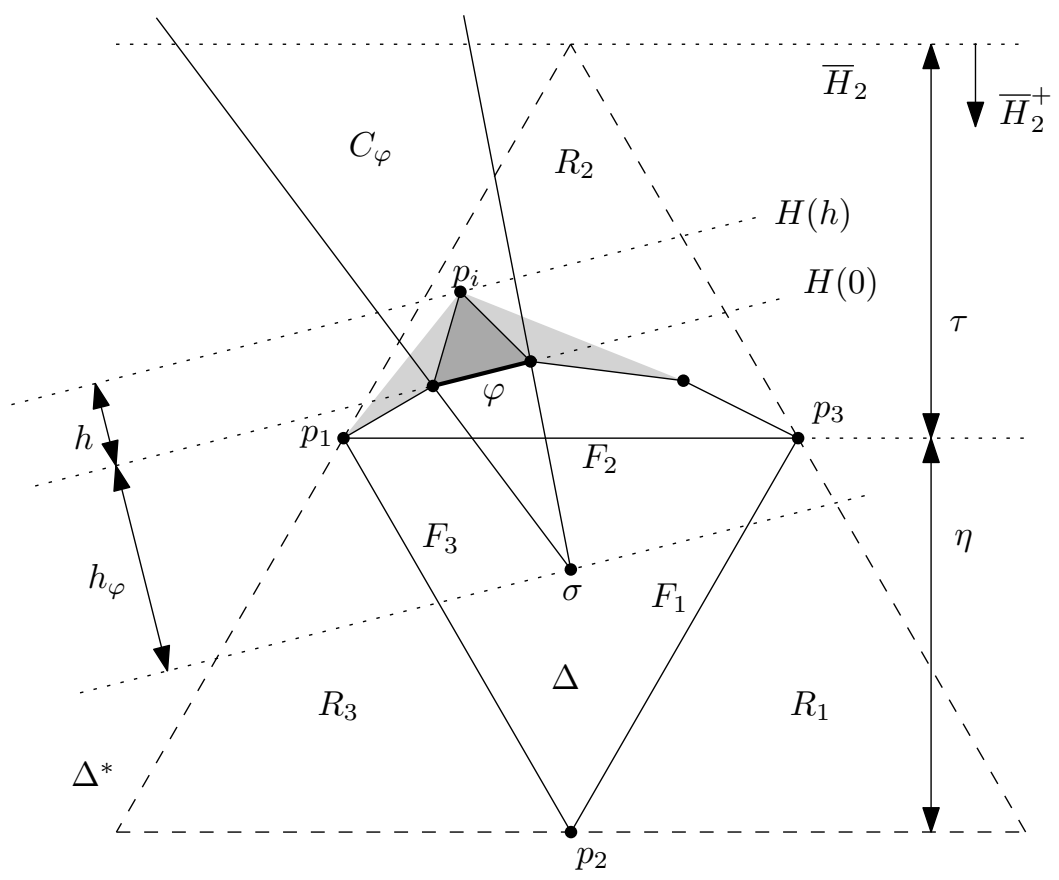

Figure 5 An illustration of the proof of Lemma 10. In order for $\left\{p_{1}, \ldots, p_{i}\right\}$ to be an $i$-island in $S$, the light gray part cannot contain points from $S$. We estimate the probability of this event from above by the probability that the dark gray $\operatorname{simplex} \operatorname{conv}\left(\varphi \cup\left\{p_{i}\right\}\right)$ contains no point of $S$. Note that the parameters $\eta$ and $\tau$ coincide for $d=2$, as then $\tau=\frac{d^{2}-1}{d+1} \eta=\eta$.

The point $p_{i}$ lies in the $(d-1)$-dimensional simplex $C_{\varphi} \cap H(h)$, which is a scaled copy of $\varphi$. We show that

$$
\lambda_{d-1}\left(C_{\varphi} \cap H(h)\right) \leq d^{2 d-2} \cdot \lambda_{d-1}(\varphi) .
$$

Let $h_{\varphi}$ be the distance between $H(0)$ and $\sigma$ and, for every $j \in\{1, \ldots, d+1\}$, let $\bar{H}_{j}$ be the hyperplane parallel to $F_{j}$ containing the vertex $H_{1} \cap \cdots \cap H_{j-1} \cap H_{j+1} \cap \cdots \cap H_{d+1}$ of $\Delta^{*}$. We denote by $\bar{H}_{j}^{+}$the halfspace determined by $\overline{H_{j}}$ containing $\Delta^{*}$. Since $\Delta$ lies on the same side of $H(0)$ as $\sigma$, we see that $h_{\varphi}$ is at least as large as the distance between $\sigma$ and $F_{j}$, which is $\eta /(d+1)$. Since $p_{i}$ lies in $\Delta^{*} \subseteq \bar{H}_{j}^{+}$, we see that $h$ is at most as large as the distance $\tau$ between $\bar{H}_{j}$ and the hyperplane containing the facet $F_{j}$ of $\Delta$. Note that $\tau+\eta /(d+1)$ is the distance of the barycenter of $\Delta^{*}$ and a vertex of $\Delta^{*}$ and $d \eta /(d+1)$ is the distance of the barycenter of $\Delta^{*}$ and a facet of $\Delta^{*}$. Thus we get $\tau=\frac{d^{2} \eta}{d+1}-\frac{\eta}{d+1}=\frac{d^{2}-1}{d+1} \eta$ from the fact that the distance between the barycenter of a $d$-dimensional simplex and any of its vertices is $d$-times as large as the distance between the barycenter and a facet. Consequently, $h \leq \frac{d^{2}-1}{d+1} \eta$ and $\frac{\eta}{d+1} \leq h_{\varphi}$, which implies $h \leq\left(d^{2}-1\right) h_{\varphi}$. Thus $C_{\varphi} \cap H(h)$ is a scaled copy of $\varphi$ by a factor of size at most $d^{2}$. This gives $\lambda_{d-1}\left(C_{\varphi} \cap H(h)\right) \leq d^{2 d-2} \cdot \lambda_{d-1}(\varphi)$.

Since the simplex $\operatorname{conv}\left(\varphi \cup\left\{p_{i}\right\}\right)$ is a subset of the closure of $\operatorname{conv}\left(\left\{p_{1}, \ldots, p_{i}\right\}\right) \backslash$ $\operatorname{conv}\left(\left\{p_{1}, \ldots, p_{i-1}\right\}\right)$, the probability $\operatorname{Pr}\left[E_{a, i} \mid E_{a, i-1}\right]$ can be bounded from above by the conditional probability of the event $A_{i, \varphi}$ that $p_{i} \in C_{\varphi} \cap K$ and that no point from $S \backslash\left\{p_{1}, \ldots, p_{i}\right\}$ lies in $\operatorname{conv}\left(\varphi \cup\left\{p_{i}\right\}\right)$, conditioned on $E_{a, i-1}$. All points from $S \backslash\left\{p_{1}, \ldots, p_{i}\right\}$ lie outside of $\operatorname{conv}\left(\varphi \cup\left\{p_{i}\right\}\right)$ with probability

$$
\left(1-\frac{\lambda_{d}\left(\operatorname{conv}\left(\varphi \cup\left\{p_{i}\right\}\right)\right)}{\lambda_{d}\left(K \backslash \operatorname{conv}\left(\left\{p_{1}, \ldots, p_{i-1}\right\}\right)\right)}\right)^{n-i} .
$$


Since $\lambda_{d}\left(K \backslash \operatorname{conv}\left(\left\{p_{1}, \ldots, p_{i-1}\right\}\right)\right) \leq \lambda_{d}(K)=1$, this is bounded from above by

$$
\left(1-\lambda_{d}\left(\operatorname{conv}\left(\varphi \cup\left\{p_{i}\right\}\right)\right)\right)^{n-i}=\left(1-\frac{\lambda_{d-1}(\varphi) \cdot h}{d}\right)^{n-i} .
$$

Since the sets $C_{\varphi}$ partition $K \backslash \operatorname{conv}\left(\left\{p_{1}, \ldots, p_{i-1}\right\}\right)$ (up to intersections of $d$-dimensional Lebesgue measure 0 ) and since $h \leq d / \lambda_{d-1}(\varphi)$, we have, by the law of total probability,

$$
\begin{aligned}
\operatorname{Pr}\left[E_{a, i} \mid E_{a, i-1}\right] & \leq \sum_{\varphi} \operatorname{Pr}\left[A_{i, \varphi} \mid E_{a, i-1}\right] \\
& \leq \sum_{\varphi} \int_{0}^{d / \lambda_{d-1}(\varphi)} \lambda_{d-1}\left(C_{\varphi} \cap H(h)\right) \cdot\left(1-\frac{\lambda_{d-1}(\varphi) \cdot h}{d}\right)^{n-i} \mathrm{~d} h .
\end{aligned}
$$

The sums in the above expression are taken over all facets $\varphi$ of the convex polytope $\operatorname{conv}\left(\left\{p_{1}, \ldots, p_{i-1}\right\}\right)$. Using (3), we can estimate $\operatorname{Pr}\left[E_{a, i} \mid E_{a, i-1}\right]$ from above by

$$
d^{2 d-2} \cdot \sum_{\varphi} \lambda_{d-1}(\varphi) \cdot \int_{0}^{d / \lambda_{d-1}(\varphi)}\left(1-\frac{\lambda_{d-1}(\varphi) \cdot h}{d}\right)^{n-i} \mathrm{~d} h .
$$

By substituting $t=\frac{\lambda_{d-1}(\varphi) \cdot h}{d}$, we can rewrite this expression as

$$
d^{2 d-2} \cdot \sum_{\varphi} \frac{d \cdot \lambda_{d-1}(\varphi)}{\lambda_{d-1}(\varphi)} \cdot \int_{0}^{1}(1-t)^{n-i} \mathrm{~d} t=d^{2 d-1} \cdot \sum_{\varphi} \int_{0}^{1} 1 \cdot(1-t)^{n-i} \mathrm{~d} t .
$$

By Lemma 7, this equals

$$
d^{2 d-1} \cdot \sum_{\varphi} \frac{0 ! \cdot(n-i) !}{(n-i+1) !}=\frac{d^{2 d-1}}{n-i+1} \sum_{\varphi} 1 .
$$

Since $\operatorname{conv}\left(\left\{p_{1}, \ldots, p_{i-1}\right\}\right)$ is a convex polytope in $\mathbb{R}^{d}$ with at most $i-1 \leq k$ vertices, Theorem 8 implies that the number of facets $\varphi$ of $\operatorname{conv}\left(\left\{p_{1}, \ldots, p_{i-1}\right\}\right)$ is at most $2\left(\begin{array}{c}k \\ \lfloor d / 2\rfloor\end{array}\right)$. Altogether, we have derived the desired bound

$$
\operatorname{Pr}\left[E_{a, i} \mid E_{a, i-1}\right] \leq \frac{2 d^{2 d-1} \cdot\left(\begin{array}{c}
k \\
\lfloor d / 2\rfloor
\end{array}\right)}{n-i+1}
$$

in the case when $\Delta$ is a regular simplex.

If $\Delta$ is not regular, we first apply a volume-preserving affine transformation $F$ that maps $\Delta$ to a regular simplex $F(\Delta)$. The simplex $F(\Delta)$ is then contained in the convex body $F(K)$ of volume 1 . Since $F$ translates the uniform distribution on $F(K)$ to the uniform distribution on $K$ and preserves holes and islands, we obtain the required upper bound also in the general case.

Now, we finish the proof of Theorem 1.

Proof of Theorem 1. We estimate the expected value of the number $X$ of $k$-islands in $S$. The number of ordered $k$-tuples of points from $S$ is $n(n-1) \cdots(n-k-1)$. Since every subset of $S$ of size $k$ admits a unique labeling that satisfies the conditions (L1), (L2), (L3), (L4), and (L5), we have 


$$
\begin{aligned}
\mathbb{E}[X] & =n(n-1) \cdots(n-k+1) \cdot \operatorname{Pr}\left[\cup_{a=0}^{k-d-1} E_{a, k}\right] \\
& =n(n-1) \cdots(n-k+1) \cdot \sum_{a=0}^{k-d-1} \operatorname{Pr}\left[E_{a, k}\right],
\end{aligned}
$$

as the events $E_{0, k}, \ldots E_{k-d-1, k}$ are pairwise disjoint.

The probability of the event $L_{2}$, which says that the points $p_{1}, \ldots, p_{d}$ satisfy the condition (L2), is $1 / d$ !. Let $P=\sum_{a=0}^{k-d-1} \operatorname{Pr}\left[E_{a, k} \mid L_{2}\right]$. For any two events $E, E^{\prime}$ with $E \supseteq E^{\prime}$ and $\operatorname{Pr}[E]>0$, we have $\operatorname{Pr}\left[E^{\prime}\right]=\operatorname{Pr}\left[E \cap E^{\prime}\right]=\operatorname{Pr}\left[E^{\prime} \mid E\right] \cdot \operatorname{Pr}[E]$. Thus, using $L_{2} \supseteq E_{a}=E_{a, d+a+1} \supseteq E_{a, d+a+2} \supseteq \cdots \supseteq E_{a, k}$, we get

$$
\mathbb{E}[X]=n(n-1) \cdots(n-k+1) \cdot \operatorname{Pr}\left[L_{2}\right] \cdot P=\frac{n(n-1) \cdots(n-k+1)}{d !} \cdot P
$$

and

$$
P=\sum_{a=0}^{k-d-1} \operatorname{Pr}\left[E_{a} \mid L_{2}\right] \cdot \prod_{i=d+a+2}^{k} \operatorname{Pr}\left[E_{a, i} \mid E_{a, i-1}\right] .
$$

For every $a \in\{d+2, \ldots, k-d-1\}$, Lemma 9 gives

$$
\operatorname{Pr}\left[E_{a} \mid L_{2}\right] \leq \frac{2^{d-1} \cdot d !}{(k-a-d-1) ! \cdot(n-k+1)^{a+1}} \leq \frac{2^{d-1} \cdot d !}{(n-k+1)^{a+1}}
$$

and, due to Lemma 10,

$$
\operatorname{Pr}\left[E_{a, i} \mid E_{a, i-1}\right] \leq \frac{2 d^{2 d-1} \cdot\left(\begin{array}{c}
k \\
\lfloor d / 2\rfloor
\end{array}\right)}{n-i+1}
$$

for every $i \in\{d+a+2, \ldots, k\}$.

Using these estimates we derive

$$
\begin{aligned}
P & \leq 2^{d-1} \cdot d ! \cdot\left(2 d^{2 d-1}\left(\begin{array}{c}
k \\
\lfloor d / 2\rfloor
\end{array}\right)\right)^{k-d-1} \cdot \sum_{a=0}^{k-d-1} \frac{1}{(n-k+1)^{a+1}} \cdot \prod_{i=d+a+2}^{k} \frac{1}{n-i+1} \\
& \leq 2^{d-1} \cdot d ! \cdot\left(2 d^{2 d-1}\left(\begin{array}{c}
k \\
\lfloor d / 2\rfloor
\end{array}\right)\right)^{k-d-1} \cdot \sum_{a=0}^{k-d-1} \frac{1}{(n-k+1)^{a+1}} \cdot \frac{1}{(n-k+1)^{k-d-a-1}} \\
& =2^{d-1} \cdot d ! \cdot\left(2 d^{2 d-1}\left(\begin{array}{c}
k \\
\lfloor d / 2\rfloor
\end{array}\right)\right)^{k-d-1} \cdot(k-d) \cdot \frac{1}{(n-k+1)^{k-d}}
\end{aligned}
$$

Thus the expected number of $k$-islands in $S$ satisfies

$$
\begin{aligned}
\mathbb{E}[X] & =\frac{n(n-1) \cdots(n-k+1)}{d !} \cdot P \\
& \leq \frac{2^{d-1} \cdot d ! \cdot\left(2 d^{2 d-1}\left(\begin{array}{c}
k \\
\lfloor d / 2\rfloor
\end{array}\right)\right)^{k-d-1} \cdot(k-d)}{d !} \cdot \frac{n(n-1) \cdots(n-k+1)}{(n-k+1)^{k-d}} \\
& =2^{d-1} \cdot\left(2 d^{2 d-1}\left(\begin{array}{c}
k \\
\lfloor d / 2\rfloor
\end{array}\right)\right)^{k-d-1} \cdot(k-d) \cdot \frac{n(n-1) \cdots(n-k+2)}{(n-k+1)^{k-d-1}} .
\end{aligned}
$$

This finishes the proof of Theorem 1. 
In the rest of the section, we sketch the proof of Theorem 2 by showing that a slight modification of the above proof yields an improved bound on the expected number $E H_{d, k}^{K}(n)$ of $k$-holes in $S$.

Sketch of the proof of Theorem 2. If $k$ points from $S$ determine a $k$-hole in $S$, then, in particular, the simplex $\Delta$ contains no points of $S$ in its interior. Therefore

$$
E H_{d, k}^{K}(n) \leq n(n-1) \cdots(n-k+1) \cdot \operatorname{Pr}\left[E_{0, k}\right] .
$$

Then we proceed exactly as in the proof of Theorem 1, but we only consider the case $a=0$. This gives the same bounds as before with the term $(k-d)$ missing and with an additional factor $\frac{1}{(k-d-1) !}$ from Lemma 9, which proves Theorem 2 .

For $d=2$ and $k=4$, Theorem 2 gives $E H_{2,4}^{K}(n) \leq 128 n^{2}+o\left(n^{2}\right)$. We can obtain an even better estimate $E H_{2,4}^{K}(n) \leq 12 n^{2}+o\left(n^{2}\right)$ in this case. First, we have only three facets $\varphi$, as they correspond to the sides of the triangle $\Delta$. Thus the term $\left(2\left(\begin{array}{c}k \\ \lfloor d / 2\rfloor\end{array}\right)\right)^{k-d-1}=8$ is replaced by 3 . Moreover, the inequality (3) can be replaced by

$$
\lambda_{1}\left(C_{\varphi} \cap H(h) \cap \Delta^{*}\right) \leq \lambda_{1}(\varphi),
$$

since every line $H(h)$ intersects $R_{j} \subseteq \Delta^{*}$ in a line segment of length at most $\lambda_{1}\left(F_{j}\right)=\lambda(\varphi)$. This then removes the factor $d^{(2 d-2)(k-d-1)}=4$.

\section{Proof of Theorem 5}

Here, for every $d$, we state the definition of a $d$-dimensional analogue of Horton sets on $n$ points from [18] and show that, for all fixed integers $d$ and $k$, every $d$-dimensional Horton set $H$ with $n$ points contains at least $\Omega\left(n^{\min \left\{2^{d-1}, k\right\}}\right) k$-islands in $H$. If $k \leq 3 \cdot 2^{d-1}$, then we show that $H$ contains at least $\Omega\left(n^{k}\right) k$-holes in $H$.

First, we need to introduce some notation. A set $Q$ of points in $\mathbb{R}^{d}$ is in strongly general position if $Q$ is in general position and, for every $i=1, \ldots, d-1$, no $(i+1)$-tuple of points from $Q$ determines an $i$-dimensional affine subspace of $\mathbb{R}^{d}$ that is parallel to the $(d-i)$ dimensional linear subspace of $\mathbb{R}^{d}$ that contains the last $d-i$ axes. Let $\pi: \mathbb{R}^{d} \rightarrow \mathbb{R}^{d-1}$ be the projection defined by $\pi\left(x_{1}, \ldots, x_{d}\right)=\left(x_{1}, \ldots, x_{d-1}\right)$. For $Q \subseteq \mathbb{R}^{d}$, we use $\pi(Q)$ to denote the set $\left\{\pi(q) \in \mathbb{R}^{d-1}: q \in Q\right\}$. If $Q$ is a set of $n$ points $q_{0}, \ldots, q_{n-1}$ from $\mathbb{R}^{d}$ in strongly general position that are ordered so that their first coordinates increase, then, for all $m \in \mathbb{N}$ and $i \in\{0,1, \ldots, m-1\}$, we define $Q_{i, m}=\left\{q_{j} \in Q: j \equiv i(\bmod m)\right\}$.

For two sets $A$ and $B$ of points from $\mathbb{R}^{d}$ with $|A|,|B| \geq d$, we say that $B$ is deep below $A$ and $A$ is high above $B$ if $B$ lies entirely below any hyperplane determined by $d$ points of $A$ and $A$ lies entirely above any hyperplane determined by $d$ points of $A$. For point sets $A^{\prime}$ and $B^{\prime}$ in $\mathbb{R}^{d}$ of arbitrarily size, we say that $B^{\prime}$ is deep below $A^{\prime}$ and $A^{\prime}$ is high above $B^{\prime}$ if there are sets $A \supseteq A^{\prime}$ and $B \supseteq B^{\prime}$ such that $|A|,|B| \geq d, B$ is deep below $A$, and $A$ is high above $B$.

Let $p_{2}<p_{3}<p_{4}<\cdots$ be the sequence of all prime numbers. That is, $p_{2}=2, p_{3}=3$, $p_{4}=5$ and so on.

We can now state the definition of the $d$-dimensional Horton sets from [18]. Every finite set of $n$ points in $\mathbb{R}$ is 1 -Horton. For $d \geq 2$, finite set $H$ of points from $\mathbb{R}^{d}$ in strongly general position is a $d$-Horton set if it satisfies the following conditions: 
(a) the set $H$ is empty or it consists of a single point, or

(b) $H$ satisfies the following three conditions:

(i) if $d>2$, then $\pi(H)$ is $(d-1)$-Horton,

(ii) for every $i \in\left\{0,1, \ldots, p_{d}-1\right\}$, the set $H_{i, p_{d}}$ is $d$-Horton,

(iii) every $I \subseteq\left\{0,1, \ldots, p_{d}-1\right\}$ with $|I| \geq 2$ can be partitioned into two nonempty subsets $J$ and $I \backslash J$ such that $\cup_{j \in J} H_{j, p_{d}}$ lies deep below $\cup_{i \in I \backslash J} H_{i, p_{d}}$.

Valtr [18] showed that such sets indeed exist and that they contain no $k$-hole with $k>2^{d-1}\left(p_{2} p_{3} \cdots p_{d}+1\right)$. The 2 -Horton sets are known as Horton sets. We show that $d$-Horton sets with $d \geq 3$ contain many $k$-islands for $k \geq d+1$ and thus cannot provide the upper bound $O\left(n^{d}\right)$ that follows from Theorem 1. This contrasts with the situation in the plane, as 2-Horton sets of $n$ points contain only $O\left(n^{2}\right) k$-islands for any fixed $k$ [8].

Let $d$ and $k$ be fixed positive integers. Assume first that $k \geq 2^{d-1}$. We want to prove that there are $\Omega\left(n^{2^{d-1}}\right) k$-islands in every $d$-Horton set $H$ with $n$ points. We proceed by induction on $d$. For $d=1$ there are $n-k+1=\Omega(n) k$-islands in every 1-Horton set.

Assume now that $d>1$ and that the statement holds for $d-1$. The $d$-Horton set $H$ consists of $p_{d} \in O(1)$ subsets $H_{i, p_{d}}$, each of size at least $\left\lfloor n / p_{d}\right\rfloor \in \Omega(n)$. The set $\left\{0, \ldots, p_{d}-1\right\}$ is ordered by a linear ordering $\prec$ such that, for all $i$ and $j$ with $i \prec j$, the set $H_{i, p_{d}}$ is deep below $H_{j, p_{d}}$; see [18]. Take two of sets $X=H_{a, p_{d}}$ and $Y=H_{b, p_{d}}$ such that $a \prec b$ are consecutive in $\prec$. Since $k \geq 2^{d-1}$, we have $\lceil k / 2\rceil \geq\lfloor k / 2\rfloor \geq 2^{d-2}$. Thus by the inductive hypothesis, the $(d-1)$-Horton set $\pi(X)$ of size at least $\Omega(n)$ contains at least $\Omega\left(n^{2^{d-2}}\right)$ $\lfloor k / 2\rfloor$-islands. Similarly, the $(d-1)$-Horton set $\pi(Y)$ of size at least $\Omega(n)$ contains at least $\Omega\left(n^{2^{d-2}}\right)\lceil k / 2\rceil$-islands.

Let $\pi(A)$ be any of the $\Omega\left(n^{2^{d-2}}\right)\lfloor k / 2\rfloor$-islands in $\pi(X)$, where $A \subseteq X$. Similarly, let $\pi(B)$ be any of the $\Omega\left(n^{2^{d-2}}\right)\lceil k / 2\rceil$-islands in $\pi(Y)$, where $B \subseteq Y$. We show that $A \cup B$ is a $k$-island in $H$. Suppose for contradiction that there is a point $x \in H \backslash(A \cup B)$ that lies in $\operatorname{conv}(A \cup B)$. Since $a$ and $b$ are consecutive in $\prec$, the point $x$ lies in $X \cup Y=H_{a, p_{d}} \cup H_{b, p_{d}}$. By symmetry, we may assume without loss of generality that $x \in X$. Since $x \notin A$ and $H$ is in strongly general position, we have $\pi(x) \in \pi(X) \backslash \pi(A)$. Using the fact that $\pi(A)$ is a $\lfloor k / 2\rfloor$-island in $\pi(X)$, we obtain $\pi(x) \notin \operatorname{conv}(\pi(A))$ and thus $x \notin \operatorname{conv}(A)$. Since $X$ is deep below $Y$, we have $x \notin \operatorname{conv}(B)$. Thus, by Carathédory's theorem, $x$ lies in the convex hull of a $(d+1)$-tuple $T \subseteq A \cup B$ that contains a point from $A$ and also a point from $B$.

Note that, for $U=(T \cup\{x\})$, we have $|U \cap A| \geq 2$, as $x \in A$ and $|T \cap A| \geq 1$. We also have $|U \cap B| \geq 2$, as $X$ is deep below $Y$ and $\pi(x) \notin \operatorname{conv}(\pi(A))$. Thus the affine hull of $U \cap A$ intersects the convex hull of $U \cap B$. Then, however, the set $U \cap A$ is not deep below the set $U \cap B$, which contradicts the fact that $X$ is deep below $Y$.

Altogether, there are at least $\Omega\left(n^{2^{d-2}}\right) \cdot \Omega\left(n^{2^{d-2}}\right)=\Omega\left(n^{2^{d-1}}\right)$ such $k$-islands $A \cup B$, which finishes the proof if $k$ is at least $2^{d-1}$. For $k<2^{d-1}$, we use an analogous argument that gives at least $\Omega\left(n^{\lfloor k / 2\rfloor}\right) \cdot \Omega\left(n^{\lceil k / 2\rceil}\right)=\Omega\left(n^{k}\right) k$-islands in the inductive step.

If $d \geq 2$ and $k \leq 3 \cdot 2^{d-1}$ then a slight modification of the above proof gives $\Omega\left(n^{\min \left\{2^{d-1}, k\right\}}\right)$ $k$-islands which are actually $k$-holes in $H$. We just use the simple fact that every 2 -Horton set with $n$ points contains $\Omega\left(n^{2}\right) k$-holes for every $k \in\{2, \ldots, 6\}$ as our inductive hypothesis. This is trivial for $k=2$ and it follows for $k \in\{3,4\}$ from the well-known fact that every set of $n$ points in $\mathbb{R}^{2}$ in general position contains at least $\Omega\left(n^{2}\right) k$-holes. For $k \in\{5,6\}$, this fact can be proved using basic properties of 2-Horton sets (we omit the details). Then we use the inductive assumption, which says that every $d$-Horton set of $n$ points contains at least $\Omega\left(n^{\min \left\{2^{d-1}, k\right\}}\right) k$-holes if $d \geq 2$ and $1 \leq k \leq 3 \cdot 2^{d-1}$. This finishes the proof of Theorem 5 . 


\section{References}

1 O. Aichholzer, M. Balko, T. Hackl, J. Kynčl, I. Parada, M. Scheucher, P. Valtr, and B. Vogtenhuber. A Superlinear Lower Bound on the Number of 5-Holes. In 33rd International Symposium on Computational Geometry (SoCG 2017), volume 77 of Leibniz International Proceedings in Informatics, pages 8:1-8:16, 2017. Full version: arXiv:1703.05253. doi:10.4230/LIPIcs.SoCG.2017.8.

2 G. E. Andrews, R. Askey, and R. Roy. Special functions, volume 71 of Encyclopedia of Mathematics and its Applications. Cambridge University Press, Cambridge, 1999. doi: 10.1017/CB09781107325937.

3 J. Balogh, H. González-Aguilar, and G. Salazar. Large convex holes in random point sets. Computational Geometry, 46(6):725-733, 2013. doi:10.1016/j.comgeo.2012.11.004.

4 I. Bárány and Z. Füredi. Empty simplices in Euclidean space. Canadian Mathematical Bulletin, 30(4):436-445, 1987. doi:10.4153/cmb-1987-064-1.

5 I. Bárány and P. Valtr. Planar point sets with a small number of empty convex polygons. Studia Scientiarum Mathematicarum Hungarica, 41(2):243-266, 2004. doi:10.1556/sscmath. 41.2004.2.4.

6 P. Erdős. Some more problems on elementary geometry. Australian Mathematical Society Gazette, 5:52-54, 1978. URL: http://www.renyi.hu/ p_erdos/1978-44.pdf.

7 L. C. Evans and R. F. Gariepy. Measure theory and fine properties of functions. Textbooks in Mathematics. CRC Press, Boca Raton, FL, revised edition, 2015.

8 R. Fabila-Monroy and C. Huemer. Covering Islands in Plane Point Sets. In Computational Geometry: XIV Spanish Meeting on Computational Geometry, EGC 2011, volume 7579 of Lecture Notes in Computer Science, pages 220-225. Springer, 2012. doi: 10.1007/978-3-642-34191-5_21.

9 R. Fabila-Monroy, C. Huemer, and D. Mitsche. Empty non-convex and convex four-gons in random point sets. Studia Scientiarum Mathematicarum Hungarica. A Quarterly of the Hungarian Academy of Sciences, 52(1):52-64, 2015. doi:10.1556/SScMath.52.2015.1.1301.

10 T. Gerken. Empty Convex Hexagons in Planar Point Sets. Discrete \& Computational Geometry, 39(1):239-272, 2008. doi:10.1007/s00454-007-9018-x.

11 H. Harborth. Konvexe Fünfecke in ebenen Punktmengen. Elemente der Mathematik, 33:116-118, 1978. In German. URL: http://www.digizeitschriften.de/dms/img/?PID= GDZPPN002079801.

12 J. D. Horton. Sets with no empty convex 7-gons. Canadian Mathematical Bulletin, 26:482-484, 1983. doi: 10.4153/CMB-1983-077-8.

13 M. Katchalski and A. Meir. On empty triangles determined by points in the plane. Acta Mathematica Hungarica, 51(3-4):323-328, 1988. doi:10.1007/BF01903339.

14 J. Matoušek. Lectures on discrete geometry, volume 212 of Graduate Texts in Mathematics. Springer-Verlag, New York, 2002. doi:10.1007/978-1-4613-0039-7.

15 M. C. Nicolas. The Empty Hexagon Theorem. Discrete \& Computational Geometry, 38(2):389397, 2007. doi:10.1007/s00454-007-1343-6.

16 Matthias Reitzner and Daniel Temesvari. Stars of empty simplices, 2019. arXiv:1808.08734.

17 M. Scheucher. Points, Lines, and Circles: Some Contributions to Combinatorial Geometry. $\mathrm{PhD}$ thesis, Technische Universität Berlin, Institut für Mathematik, 2019.

18 P. Valtr. Sets in $\mathbb{R}^{d}$ with no large empty convex subsets. Discrete Mathematics, 108(1):115-124, 1992. doi : 10.1016/0012-365X (92) 90665-3.

19 P. Valtr. On the minimum number of empty polygons in planar point sets. Studia Scientiarum Mathematicarum Hungarica, pages 155-163, 1995. URL: https://refubium.fu-berlin.de/ handle/fub188/18741.

20 P. Valtr. On empty hexagons. In Surveys on Discrete and Computational Geometry: Twenty Years Later, volume 453 of Contemporary Mathematics, pages 433-441. American Mathematical Society, 2008. URL: http://bookstore.ams.org/conm- 453.

21 P. Valtr. On empty pentagons and hexagons in planar point sets. In Proceedings of Computing: The Eighteenth Australasian Theory Symposium (CATS 2012), pages 47-48, Melbourne, Australia, 2012. URL: http://crpit.com/confpapers/CRPITV128Valtr.pdf. 